\title{
T7Max transcription system
}

Christopher Deich ${ }^{1}$, Brock Cash ${ }^{1}$, Wakana Sato ${ }^{1}$, Judee Sharon ${ }^{1}$, Lauren Aufdembrink ${ }^{1}$, Nathaniel J. Gaut ${ }^{1}$, Joseph Heili ${ }^{1}$, Kaitlin Stokes ${ }^{1}$, Aaron E Engelhart ${ }^{1 *}$, Katarzyna P Adamala ${ }^{1 *}$

${ }^{1}$ Department of Genetics, Cell Biology and Development, University of Minnesota, Minneapolis, MN US. *Correspondence to enge0213@umn.edu and kadamala@umn.edu

\begin{abstract}
Efficient cell-free protein expression from linear DNA templates has remained a challenge primarily due to template degradation. Here we present a modified T7 RNA polymerase promoter that acts to significantly increase the yields of both transcription and translation within in vitro systems. The modified promoter, termed T7Max, recruits standard T7 RNA polymerase, so no protein engineering is needed to take advantage of this method. This technique could be used with any T7 RNA polymerasebased in vitro protein expression system. Unlike other methods of limiting linear template degradation, the T7Max promoter increases transcript concentration in a T7 transcription reaction, providing more mRNA for translation.
\end{abstract}

Keywords: in vitro transcription, in vitro translation, synthetic cells, cell-free protein expression

\section{Introduction}

The T7 promoter for the RNA polymerase of bacteriophage T7 consists of 18 base pairs of sequence $\left(5^{\prime}-\right.$ TAATACGACTCACTATAG - 3').[1] Previous research identified the relationship between the sequence and transcriptional efficiency, which helped to strengthen the T7 system's usability.[2-4] 
Due to the T7 system's versatility, the T7 system can be used both in vivo and in a cell-free translation system (CFTS). For example, bacterial cell-free translation systems commonly use the T7 RNA polymerase alongside the endogenous sigma 70 system.[5] Furthermore, cell-free translation system platforms derived from hosts other than bacteria are also coupled with the T7 transcription, like plant[6], mammalian[7], and insect[8] in vitro translation systems.

We investigated whether translation in a cell free transcription - translation system (TxTI) can be increased by improving the availability of the mRNA. It has been shown that increasing plasmid concentration directly correlates with increased translation yields in bacterial TxTI.[9] We reasoned that increasing the abundance of mRNA, with all other components of the translation system being equal, should result in both an increase of protein abundance and an increased resistance to DNA template degradation by endogenous nucleases in TxTI.

\section{Results and discussion}

Due to the robustness and high popularity of T7 RNA polymerase, there has been a lot of effort in engineering T7 RNA polymerase promoter sequences. $[3,10]$ We began by investigating efficiency of several known T7 promoter variants (Table 1). [11,12] We constructed double stranded linear DNA templates coding for the broccoli fluorescent RNA aptamer [13] with each of the tested T7 promoter variants. The templates had no terminators, so all transcriptions were run-off terminated.

Each transcription reaction was analyzed on a urea PAGE gel with the product stained with DFHBI1T, the ligand for the broccoli aptamer. The resulting image shows only correctly folded full length broccoli aptamer products (Figure 1a). The gel was then de-stained and stained again using the general nucleic acid stain Sybr Gold. This stain shows all nucleic acid present in the sample, including truncation products of transcription (Figure 1c). Both DFHBI and Sybr stained gels were quantified, comparing the 
relative abundance of the full-length broccoli aptamer product to the total nucleic acid abundance in the sample (Figure $\mathbf{1 b}$ and $\mathbf{1 d}$ ).

We identified two promoters (Uhlenbeck GG and Uhlenbeck 117) that show the highest yields of fluorescent RNA product. We also performed time course fluorescent readout of transcription from all the tested promoters, measuring transcription fluorescence for 6 hours (Figure 1e, and end point quantification shown on panel 1f). We selected the two most promising promoters for further tests: Uhlenbeck GG and Uhlenbeck 117.

Next, we proceeded to test full translation efficiency, still using linear dsDNA template.

We constructed eGFP templates with each of the tested promoters, using UTR1 and T500 terminator sequences optimized for bacterial in vitro translation. [5] The translation efficiency was measured by fluorescence of eGFP after an 8 hour reaction (Figure 2a). We quantified the abundance of eGFP mRNA using RT qPCR (Figure $\mathbf{2 b}$ ). One of the promoters provided a slightly higher protein product amount, and higher mRNA abundance. That promoter, with the sequence AATTCTAATACGACTCACTATAGGGA, which we named "T7Max" - is an improved T7 promoter variant.

Escherichia coli has many endogenous DNA nucleases[14], which make their way into the TxTI extract without losing activity[15] and thus cause degradation of linear DNA templates in TxTI. Several methods have been proposed for enabling linear template expression, mainly focused on blocking the activity of the $\operatorname{RecBCD}$, one of the more well-characterized nucleases. Among those methods, the most popular are the addition of GamS protein[16] or small DNA Chi6 [17] - both inhibiting RecBCD nuclease.

We used both the Chi6 inhibitors, and the GamS protein inhibitor. We tested expression of eGFP under classic T7 and under T7Max, from the same linear templates described above, using either E. coli extract made in our lab, or MyTXTL, a commercial E. coli TxTl extract from Arbor Biosciences (Figure 2c). All 
reactions were set up with identical DNA template concentrations and in each compared pair (T7 vs T7Max) all other conditions, like concentration of RecBCD inhibitor, were the same. In all cases, the T7Max promoter outperformed the classic T7 promoter, as measured by GFP fluorescence after an 8 hour reaction. In some cases, expression under the T7Max promoter was 5 times larger than expression under the classic T7 promoter (in cases of GamS experiments, Figure $\mathbf{2 c}$ ). In addition to fluorescence measurements, we confirmed via a Western Blot one sample for each of the tested conditions (Figure 2d).

After establishing that the T7Max promoter outperforms the classic T7 promoter in expression from linear DNA templates, we moved on to further characterizing the T7Max promoter in translation reactions.

We used two circular DNA plasmids using UTR1 and T500 terminator and eGFP, identical except for the sequence of the promoter. First, we compared the kinetics of eGFP translation in E. c oli TxTI (Figure 3a), and corresponding GFP mRNA abundance (Figure 3c).

The T7Max promoter consistently provided a higher level of fluorescence and a higher copy number of mRNA than the classic T7 promoter. To ensure that the measured protein abundance is not a fluorescence artifact, we analyzed eGFP abundance via Western Blot (Figure 3b) and then quantified the Western Blot gels (Figure 3d). The T7Max promoter consistently produced higher protein abundance.

To further characterize performance of the T7Max promoter in cell-free protein expression reactions, we analyzed reactions at different temperatures. In addition to $30^{\circ} \mathrm{C}$ (the optimal E. c oli $\mathrm{TxTI}$ reaction temperature used throughout this paper), we analyzed reactions at $25^{\circ} \mathrm{C}$ and $37^{\circ} \mathrm{C}$ (Figure $4 \mathrm{a}$ ).

In all cases, T7Max produced more protein product, confirmed by RT qPCR measurements of mRNA abundance (Figure $\mathbf{4 b}$ ). The advantage of T7Max was largest at $30^{\circ} \mathrm{C}$, the optimal TxTI temperature, 
however the qPCR data shows significantly higher abundance of mRNA produced from T7Max vs classic

$\mathrm{T} 7$ at $37^{\circ} \mathrm{C}$ as well. We speculate that this discrepancy might be due to the generally decreased translation performance at higher temperatures.

The analysis of mRNA abundance in a TxTI reaction over time (Figure 4c) demonstrates that T7Max consistently produces more mRNA than classic T7, however the biggest difference is visible at the 2-hour mark. We speculate this might be due to the interplay between mRNA synthesis and degradation: the mRNA produced from the T7Max promoter is identical to the one produced from the classic T7 promoter, therefore after significantly higher accumulation initially due to faster transcription, the abundance evens out due to similar levels of mRNA degradation.

We also investigated the influence of the T7 RNA polymerase concentration on translation performance (Figure 4d). Comparing the T7Max promoter with the classic T7 promoter demonstrates that the T7Max promoter produces higher protein yield at higher T7 RNA polymerase concentrations. However, as the T7 RNA polymerase concentration decreases, the difference between the T7Max and classic T7 templates starts to even out. We speculate this is because at lower RNA polymerase concentrations, the polymerase concentration becomes the rate limiting factor. While T7Max provides more efficient translation, if there is not enough polymerase to bind to all DNA templates, the promoter strength becomes less significant.

To thoroughly characterize the difference in T7Max performance vs classic T7 performance, we expressed several different types of proteins differing in open reading frame size from $1650 \mathrm{bp}$ to $30 \mathrm{bp}$ (Figure 5).

We expressed luciferases: firefly (Fig. 5a), Renilla (Fig. 5d) and Nanoluc[18] (Fig. 5f). We expressed viral coat protein RNA binding proteins PP7[19] (Fig. 5g) and MS2[20] (Fig. 5h). We expressed the protein 
OphA from Omphalotus olearius Jack-o'-Lantern mushroom (Fig. 5b). We expressed the DNA restriction enzyme EcoRI (Fig. 5c), and the kanamycin resistance protein (Fig. 5e). We also expressed the extremely small fluorescent protein aptamer, FIAsH aptamer, which binds an arsenic ligand[21] (Fig. 5i). Thus, we covered a wide range of protein sizes, and many possible mRNA folds.

In all cases, in addition to measuring the protein abundance after an 8 hour TxTI reaction, we performed RT qPCR analysis of mRNA abundance. In all cases, T7Max templates produced more protein and higher mRNA abundance than classic T7 templates.

Cell-free translation systems are key components of most synthetic minimal cell designs.[23] We tested the T7Max promoter in the cytoplasm of a synthetic cell : encapsulating E. c oli TxTI in POPC / cholesterol liposomes.[22] We prepared samples of synthetic cells with phospholipid membranes, dyed red with Rhodamine-PE dye, and bacterial TxTI with eGFP -encoding plasmid under the control of either the classic T7 promoter or our T7Max promoter. (Figure 6). Imaging of the diluted samples clearly showed individual synthetic cell liposomes expressing GFP in the lumen (Figure 6a and 6b). To increase the number of samples analyzed in each field of view, we also imaged undiluted samples, at higher concentrations of lipids (Figure $6 c$ and $\mathbf{6 d}$ ). We quantified fluorescence from these images, measuring total fluorescence in the GFP channel to estimate protein production and then normalizing that value to total fluorescence in the red channel ( normalizing to the number of liposomes in each field of view). Synthetic cells expressing GFP under the T7Max promoter showed higher protein production than synthetic cells containing the classic T7 promoted GFP.

Next, we asked how will T7Max compare to classic T7 in other in vitro translation systems. Other in vitro translation systems are used for different applications[23,24], including the PURE system composed of $E$. coli translation machinery purified individually[25], wheat germ extract[26], Leishmania tarentolae 
extract[27], insect Spodoptera frugiperda Sf21 cell line extract[28], and rabbit reticulocyte extract[29].

All of those extracts are commercially available and were used according to the manufacturer's protocols.

We created templates for eGFP expression in each of those cell-free systems, with the only difference between templates being the T7 RNA polymerase promoter: either T7Max or classic T7.

Because the absolute yields (measured as GFP fluorescence) were different in each extract, we normalized the results: the classic T7 promoter is assigned value 100, and the T7Max template fluorescence is proportionally scaled for each sample. For example, the raw fluorescence value for classic T7 promoter E. coli in this case was 9384, while T7Max value was 15671; normalizing T7 to 100 gives T7Max value of 167 (Figure 7a). In all tested cases, the yield of protein synthesis was higher from a template using the T7Max promoter than from the template using the classic T7 promoter.

Finally, we looked to other applications for T7Max. Robust, sensitive, and transportable disease detection systems are in great need, and many rely on the amplification of nucleic acids.[30] AptaNASBA is an isothermal exponential disease detection reaction, dependent on the productivity of T7 RNA polymerase.[31] In Apta-NASBA, primers introduce the T7 RNA polymerase promoter and result in a fluorescent read out via an RNA aptamer. We designed primers to detect the aggR gene of $E$. Colione incorporating T7Max and the other, classic T7. All other reaction components were kept identical. Reactions where T7Max was incorporated created a 14X signal compared to a negative control (a reaction lacking template) vs $1.24 \mathrm{X}$ when incorporating classic T7 after 100 minutes (Figure7b). Such increase in signal can allow for a more sensitive detection reaction.

Cell free expression platforms find increasingly versatile applications in many areas of bioengineering, synthetic biology, and metabolic engineering. [32-34] Additionally, the focus for engineering synthetic minimal cells is on reconstituting in vitro translation reactions, most often with the use of a bacterial 
translation system and T7 RNA polymerase.[35,36] Here we demonstrated a simple technique to enable a significant increase in translation yield via a change of the T7 promoter sequence.

This system utilizes all existing elements of T7 RNA polymerase- driven transcription without changes and only requires replacement of the promoter sequence in the construct.

We have demonstrated versatile utility of the T7Max promoter in multiple different cell-free protein expression systems and for proteins over a wide range of sizes and types, as well as significantly increased yields of protein synthesis from linear DNA templates.

With the production of mRNA vaccines, there has been a recent increase of significance for in vitro transcription, in particular with the use of the T7 RNA polymerase.[37,38] This increases the need for optimizing transcription reactions. While the sequence of the T7Max promoter has been known for decades, this is the first comprehensive characterization of its use for in vitro transcription and translation. Our hope is this technology will enable further improvements in both transcription and in vitro protein expression to result in better biomedical, biotechnological and synthetic cell engineering applications.

\section{Materials and methods}

Construction of Tx Templates for Screen of Different Promoters

A series of T7 promoters described previously [1,2,11,12], see Table 1 for sequences, were placed upstream of the broccoli coding sequence via primer extension. Sense strand primers with promoter sequences, the first 23 nucleotides of the broccoli coding sequence, and the anti-sense primers coding for broccoli (49 nucleotides) were synthesized (Integrated DNA Technologies) and brought to $10 \mu \mathrm{M}$ in 
Millipore water (GenPure Pro UV-TOC/UF). Bulldog Bio BioReady Taq DNA Polymerase (BSA12L010) was used according to manufacturer's instructions with NEB dNTPs (N0446S). $9 \mu \mathrm{L}$ of PCR master mix reagents and anti-sense primer were combined with $1 \mu \mathrm{L}$ of the promoter primer to give a primerextension reaction of $1 \mathrm{X}$ Bulldog Reaction Buffer, $1 \mu \mathrm{M}$ of both primers, $1 \mathrm{mM}$ dNTPs, BioReady rTaq $(0.05 \mathrm{U} / \mu \mathrm{L})$. The reaction was denatured for 5 seconds at $95^{\circ} \mathrm{C}$, annealed for 5 minutes at $60^{\circ} \mathrm{C}$, and then extended for $30 \mathrm{~min}$ at $72^{\circ} \mathrm{C}$ (Bio-Rad T100 thermocycler). These reactions were generated in triplicate for each promoter tested, which served as a 10X stock of template in a transcription reaction.

\section{Transcription}

The templates were then used as -is in a transcription assay. All reagents, tubes, and plates were prechilled on ice. A master mix of transcription reagents was prepared on ice, and $9 \mu \mathrm{L}$ of the master mix and $1 \mu \mathrm{L}$ of the $10 \mathrm{X}$ templates were combined in a $200 \mu \mathrm{L}$ PCR tube, flicked, spun down, and then transferred to a cold, clear bottom 384-well plate. The transcriptions (1X template, $1 \mathrm{X}$ Homemade NEB Buffer, 8 mM GTP, 4 mM A/C/UTP, 0.005X phosphatase 25 ng/ $\mu$ L, $1 \mu$ M T7 RNAP, $100 \mu$ M DFHBI-1T, RNAse inhibitor $0.4 \mathrm{U} / \mu \mathrm{L}$ ) were incubated for 6 hours at $37^{\circ} \mathrm{C}$ in a SpectraMax Gemini XS microplate fluorimeter and data collected every 5 minutes (excitation: $472 \mathrm{~nm}$, emission: $507 \mathrm{~nm}$ ). An endpoint measurement was taken and the transcriptions stored at $-80^{\circ} \mathrm{C}$.

The fluorescent data was correlated by resolving the transcriptions in a denaturing polyacrylamide gel. An 8M urea, 10\% (19:1) PAGE was prerun for 30 minutes at 100V in a Mini PROTEAN tank (Bio-Rad) electrophoresis chamber using 1X TBE (89 mM Tris, 89 mM boric acid, 2 mM EDTA, pH 8.0). Transcriptions were diluted 1:1 with 2x TBE Loading Buffer ( $8 \mathrm{M}$ urea, $89 \mathrm{mM}$ Tris, $89 \mathrm{mM}$ boric acid, 2 mM EDTA, pH 8.0) and the entire $20 \mu \mathrm{L}$ sample was resolved for 1 hour at $125 \mathrm{~V}$. The gel was then equilibrated in $50 \mathrm{~mL} 1 \mathrm{X}$ folding buffer (1 mM MgCl2, $50 \mathrm{mM} \mathrm{KCl}, 10 \mathrm{mM}$ Tris, $\mathrm{pH} \mathrm{8.0)} \mathrm{for} 45$ minutes. The buffer was then decanted, exchanged with $50 \mathrm{~mL} 1 \mathrm{X}$ folding buffer supplemented with $10 \mu \mathrm{M}$ 
DFHBI-1T, and incubated for 15 minutes at room temperature. The broccoli band was imaged on an Aplegen Omega Lum G using a SYBR Safe filter. The buffer was decanted as before and replaced with $1 X$ Folding Buffer supplemented with 1X SYBR Gold (Thermo Scientific, S11494). After a 15 minute incubation at room temperature, the total RNA was imaged using the aforementioned filter. Low range ssRNA Ladder (New England BioLabs, Cat no N0364S) and 10 pmol of broccoli were run alongside the transcriptions as controls. The RNA produced for both stain s was quantified using GelQuant.NET.

\section{Construction of T7Max Plasmids}

Double stranded T7Max promoter insert was formed from a pair of annealed 5'-phosphorylated primers. Primers were designed with 4 bp 5' overhangs just upstream of a restriction enzyme digestion site, the forward primer containing the Agel restriction site and the reverse primer containing the BgllI restriction site, using Geneious 7.1.9 (https://www.geneious.com/) and purchased from IDT. For the promoter insert primers, the forward primer sequence was 5'-

/5Phos/GATCTAATTCTAATACGACTCACTATAGGGAAATAATTTTGTTTAACTTTAAGAA-3' and the reverse primer sequence was $5^{\prime}-$

/5Phos/CCGgtATATCTCCTTCTTAAAGTTAAACAAAATTATTTCCCTATAGTGAGTCGTA-3'. The T7 promoter sequence was excised from the original plasmid backbone, UTR1-T7RNAP-T500 (Catalog No. 67739, Addgene), via restriction digestion with Agel and BgllI. The T7Max promoter was cloned into backbones containing the genes for eGFP, fluorescein arsenical hairpin (FIAsH) peptide, and Omphalotin A (OphA) by following NEB's restriction digest protocol (NEB \#R0744), 5' dephosphorylation protocol (NEB \#M0289) and T4 DNA ligase protocol (NEB \#M0202). Ligated constructs were transformed into the E. c oli strain BL21(DE3) and plated on LB agar plates containing $100 \mu \mathrm{g} / \mathrm{ml}$ carbenicillin. Colony constructs were verified by sequencing. 


\section{Western Blot}

C-terminus $6 x$ His -tagged proteins were expressed in v itro with transcription-translationally active E. c oli cell-free extract using the protocol described before[39]. Constructs were expressed for 8 hours at $30^{\circ} \mathrm{C}$ using a Bio-Rad T100 thermo cycler running software version 1.201. Samples were mixed 1:1 with 2X SDS loading buffer (100 mM Tris HCl, 2.5\% SDS, 20\% Glycerol, 4\% Beta -mercaptoethanol, 0.1\% Bromophenol Blue). Mixtures of loading buffer and sample were boiled at $95^{\circ} \mathrm{C}$ for 5 minutes in a BioRad T100 thermo cycler. Boiled samples were fractionated on a 37.5:1 Acrylamide:Bis-Acrylamide SDSPage gel and then transferred to a $0.2 \mu \mathrm{m}$ nitrocellulose membrane using a Mini-PROTEAN tank (BioRad) according to the manufacturer's protocol. Gels were run for 60 minutes at $100 \mathrm{~V}$ in $800 \mathrm{~mL}$ of $1 \mathrm{X}$ SDS running buffer (25mM Tris, $192 \mathrm{mM}$ Glycine, 3.5mM SDS). Gels were transferred for 60 minutes at $100 \mathrm{~V}$ in $1 \mathrm{~L}$ of $1 \mathrm{X}$ transfer buffer (25mM Tris, $192 \mathrm{mM}$ Glycine). Electrical current was provided by Bio-Rad Power Pac 3000. Membrane was incubated with 5\% nonfat milk in TBST (20mM Tris, pH 7.4, 150mM $\mathrm{NaCl}, 0.05 \%$ tween) for 60 minutes on a horizontal rocker (Benchmark) before mouse IgG1 anti-his primary antibodies (1:5000), purchased from Biolegend, were added to the solution. The $5 \%$ nonfat milk TBST and mouse IgG1 mixture incubated with the membrane for 60 minutes on a horizontal rocker. After incubation with primary antibodies, the membrane was rinsed three times with TBST followed by three 10 min washes in TBST. The membrane was next added to $5 \%$ nonfat milk in TBST containing horseradish peroxidase-conjugate goat anti-mouse IgG1 secondary antibodies (Biolegend 405306) diluted at 1:5000 and incubated on a horizontal rocker for 60 minutes. After incubation with secondary antibodies, the membrane was rinsed three times with TBST followed by three 10 minute washes in TBST. Blots were developed with SuperSignal (Thermo Scientific) immunoblotting detection system according to manufacturer's protocols. Blots were imaged using the ChemiDoc MP Imaging System (BioRad) running Image Lab version 5.2.1. 
Measuring promoter-dependent protein expression using cell-free TXTL

To prepare the E. coli cell extract and TXTL master mix, we followed the protocol outlined by Sun et al.[39]. The eGFP, fluorescein arsenical hairpin (FIAsH) peptide, or Omphalotin A (OphA) genes with Cterminal His-tags were cloned into the UTR1-T7RNAP-T500 plasmid backbone (Catalog No. 67739, Addgene). The T7 Max promoter was further cloned into these plasmids for downstream experiments. The linear version of the eGFP plasmid was created through restriction enzyme digestion of the circular plasmid with BamHI. To measure the differences in protein expression between the two promoters, $10 \mathrm{nM}$ of templates with each promoter type were added to TXTL reactions and incubated at $30^{\circ} \mathrm{C}$ for 8 hours (T100 Thermal Cycler, Bio-Rad). Post-incubation, protein expression was determined through measurement of fluorescence (eGFP and FIAsH) or Western Blot (OphA). eGFP fluorescence was standardized to $1 \mu \mathrm{M}$ fluorescein.

FIAsH peptide expression was determined through the addition of $5 \mu \mathrm{M}$ FIAsH dye and 20mM 2-(Nmorpholino)ethanesulfonic acid (MES) buffer and were standardized to samples without the peptide. The excitation and emission spectra of FIAsH intersects with that of Chai Green Dye 20X (Catalog No. R01200, Chai Bio) in the subsequent quantitative polymerase chain reaction experiments, so $10 \mu \mathrm{L}$ of the peptide's TXTL reactions were saved for transcript quantification prior to determining expression levels.

Relative comparison of transcripts with Reverse Transcription-quantitative Polymerase Chain Reaction (RT-qPCR)

Template DNA in $10 \mu \mathrm{L}$ of the TXTL reaction was degraded by adding $0.5 \mu \mathrm{L}$ of TURBO DNase $(2 \mathrm{U} / \mu \mathrm{L}$, Catalog No. AM2238, Invitrogen). The mixture was incubated at $37^{\circ} \mathrm{C}$ for 30 minutes. The enzyme and the expressed proteins were inactivated by adding 15mM EDTA (Catalog No. E9884, Sigma-Aldrich) at 
$75^{\circ} \mathrm{C}$ for 10 minutes (T100 Thermal Cycler, Bio-Rad). The denatured proteins were pelleted through centrifugation at $3,200 \mathrm{~g}$ for 2 minutes.

Forward and reverse primers (Integrated DNA Technologies), for each protein sample were created for downstream reverse transcription and qPCR experiments. Each primer pair was compatible for transcripts produced from the old promoter and T7 Max. For eGFP, the forward primer was 5'AAGTTCATCTGCACCACC-3' and the reverse primer was 5'-TTGAAGTCGATGCCCTTC-3'. For the FIAsH peptide, the forward primer was 5'-TATACCGGTATGTGGGACTG-3' and the reverse primer was 5'GATGGTGATGATGGTGATGG-3'. For OphA, the forward primer was 5'-ACGACAATGGCAAGTCCA-3' and the reverse primer was 5'-GGAAATCCGATGCCTCGT-3'.

To prepare the reverse transcription reaction, $2 \mu \mathrm{L}$ of the DNase-treated sample was mixed with $2 \mu \mathrm{L}$ of $10 \mu \mathrm{M}$ reverse primer, $4 \mu \mathrm{L}$ of $5 X$ Protoscript II Reverse Transcriptase Buffer, $1 \mu \mathrm{L}$ of Protoscript II Reverse Transcriptase (200U/ $\mu$ L, Catalog No. M0368, New England BioLabs Inc.), $2 \mu$ L of 0.1M dithiothreitol (DTT), $1 \mu \mathrm{L}$ of $10 \mathrm{mM}$ dNTP, $0.2 \mu \mathrm{L}$ of RNase Inhibitor (Catalog No. M0314, New England BioLabs Inc.), and $8 \mu \mathrm{L}$ of nuclease-free water. The reverse transcription reaction was incubated at $42^{\circ} \mathrm{C}$ for 1 hour and the reverse transcriptase was inactivated at $65^{\circ} \mathrm{C}$ for 20 minutes.

The quantitative PCR reaction mix was prepared by mixing $2 \mu \mathrm{L}$ of complementary DNA from the reverse transcription with $2 \mu \mathrm{L}$ of $10 \mu \mathrm{M}$ forward and reverse primers, $11.25 \mu \mathrm{L}$ OneTaq Hot Start $2 \mathrm{X}$ Master Mix with Standard Buffer (Catalog No. M0484, New England BioLabs Inc.), 1.25 $\mu$ L Chai Green Dye 20X (Catalog No. R01200, Chai Bio), and 7.5 $\mu$ L of nuclease-free water. The qPCR was completed using Open qPCR (Chai Biotechnologies) with the following thermocycling program: 1 cycle of 30 second denaturation at $95^{\circ} \mathrm{C}, 30$ cycles of 15 second denaturation at $95^{\circ} \mathrm{C}, 15$ second annealing at $50^{\circ} \mathrm{C}, 1$ minute extension at $68^{\circ} \mathrm{C}$, and 1 cycle of 5 -minute final extension at $68^{\circ} \mathrm{C}$. The amplification curves plotted through the Open qPCR software to determine Cq values and averages across 3 replicates of each promoter type were calculated separately. 
For experiments involving the kinetic determination of protein expression and transcript comparison, 50 $\mu \mathrm{L}$ of TXTL reactions with $10 \mathrm{nM}$ DNA templates were incubated at $30^{\circ} \mathrm{C}$ for 8 hours. Every 2 hours, including at the start of the incubation, $10 \mu \mathrm{L}$ samples were removed to measure protein expression and quantify transcription.

Apta-NASBA reactions

Apta-NASBA reactions were performed as previously described.[31] Primers used for the Apta-NASBA reaction were: Broccoli aptamer coding primer (broccoli is in italics) 5'-

GAGCCCACACTCTACTCGACAGATACGAATATCTGGACCCGACCGTCTCCAGCGATACATTAAGACGCCTAAAG-3' classic T7 primer (promoter is in italics) 5'-TAATACGACTCACTATAGCGTCAGCATCAGCTACAATTATTCC-3' T7Max primer (promoter is in italics) 5'-

\section{AATTCTAATACGACTCACTATAGGGAGACGTCAGCATCAGCTACAATTATTCC-3'}

\section{Acknowledgments}

We thank Richard Murray for providing a sample of the GamS protein. We thank Vincent Noireaux for advice on T7 RNAP expression and on using Chi6 inhibitor system. We thank Michael Freeman for the gift of OphA protein from Omphalotus olearius Jack-o'-Lantern mushroom.

This work was supported by the NIH award 5R01MH114031, RNA Scaffolds for Cell Specific Multiplexed Neural Observation; NSF award 1840301, RoL:FELS:RAISE: Building and Modeling Synthetic Bacterial Cells; and John Templeton Foundation award 61184, Exploring the Informational Transitions Bridging Inorganic Chemistry and Minimal Life.

Authors' contributions: CD, BC, JS, WS, NG, JH, KS and KA performed protein expression experiments. CD, JS, WS and KA wrote the manuscript. All authors analyzed data, edited and approved the manuscript. 
Availability of data and materials: None.

Consent for publication: All authors agree with publishing this article within The Journal of Biological

Engineering

Competing interests: The authors declare no competing interests with the publishing of this article

\section{Literature}

1. Rong M, He B, McAllister WT, Durbin RK. Promoter specificity determinants of T7 RNA polymerase.

Proc Natl Acad Sci. 1998;95:515-9.

2. Ikeda RA, Warshamana GS, Chang LL. In Vivo and in Vitro Activities of Point Mutants of the Bacteriophage T7 RNA Polymerase Promoter. Biochemistry. 1992;31:9073-80.

3. Komura R, Aoki W, Motone K, Satomura A, Ueda M. High-throughput evaluation of T7 promoter variants using biased randomization and DNA barcoding. Isalan M, editor. PLoS One. 2018;13:e0196905. 4. Nie Z, Luo H, Li J, Sun H, Xiao Y, Jia R, et al. High-Throughput Screening of T7 Promoter Mutants for Soluble Expression of Cephalosporin C Acylase in E. coli. Appl Biochem Biotechnol. Applied Biochemistry and Biotechnology; 2020;190:293-304.

5. Garamella J, Marshall R, Rustad M, Noireaux V. The all E. coli TX-TL Toolbox 2.0: a platform for cellfree synthetic biology. ACS Synth Biol [Internet]. 2016;5:344-55. Available from: http://pubs.acs.org/doi/abs/10.1021/acssynbio.5b00296

6. Madin K, Sawasaki T, Ogasawara T, Endo Y. A highly efficient and robust cell-free protein synthesis system prepared from wheat embryos: Plants apparently contain a suicide system directed at 
ribosomes. Proc Natl Acad Sci. 2000;97:559-64.

7. Wang S, Majumder S, Emery NJ, Liu AP. Simultaneous monitoring of transcription and translation in mammalian cell-free expression in bulk and in cell-sized droplets. Synth Biol. 2018;3:1-9.

8. Stech M, Quast RB, Sachse R, Schulze C, Wüstenhagen Stefan Kubick DA. A continuous-exchange cellfree protein synthesis system based on extracts from cultured insect cells. PLoS One. 2014;9.

9. Shin J, Noireaux V. Efficient cell-free expression with the endogenous E. Coli RNA polymerase and sigma factor 70. J Biol Eng. 2010;4:8.

10. Conrad T, Plumbom I, Alcobendas M, Vidal R, Sauer S. Maximizing transcription of nucleic acids with efficient T7 promoters. Commun Biol 202031 [Internet]. Nature Publishing Group; 2020 [cited 2021 Oct 5];3:1-8. Available from: https://www.nature.com/articles/s42003-020-01167-x

11. Milligan JF, Groebe DR, Witherell GW, Uhlenbeck OC. Oligoribonucleotide synthesis using T7 RNA polymerase and synthetic DNA templates. Nucleic Acids Res. 1987;15:8783-98.

12. Paul S, Stang A, Lennartz K, Tenbusch M, Überla K. Selection of a T7 promoter mutant with enhanced in vitro activity by a novel multi-copy bead display approach for in vitro evolution. Nucleic Acids Res. 2013;41.

13. Paige JS, Wu KY, Jaffrey SR. RNA mimics of green fluorescent protein. Science (80- ). 2011;333:642-6. 14. Lovett ST. The DNA Exonucleases of Escherichia coli. EcoSal Plus. 2011;4.

15. Michel-Reydellet N, Woodrow K, Swartz J. Increasing PCR fragment stability and protein yields in a cell-free system with genetically modified Escherichia coli extracts. J Mol Microbiol Biotechnol. $2005 ; 9: 26-34$.

16. Sun ZZ, Yeung E, Hayes CA, Noireaux V, Murray RM. Linear DNA for rapid prototyping of synthetic biological circuits in an escherichia coli based TX-TL cell-free system. ACS Synth Biol. 2014;3:387-97. 17. Marshall R, Maxwell CS, Collins SP, Beisel CL, Noireaux V. Short DNA containing $\chi$ sites enhances DNA stability and gene expression in E. coli cell-free transcription-translation systems. Biotechnol Bioeng. 
2017;114:2137-41.

18. Hall MP, Unch J, Binkowski BF, Valley MP, Butler BL, Wood MG, et al. Engineered luciferase reporter from a deep sea shrimp utilizing a novel imidazopyrazinone substrate. ACS Chem Biol. 2012;7:1848-57. 19. Lim F, Peabody DS. RNA recognition site of PP7 coat protein. Nucleic Acids Res. 2002;30:4138-44. 20. Parrott a M, Lago H, Adams CJ, Ashcroft a E, Stonehouse NJ, Stockley PG. RNA aptamers for the MS2 bacteriophage coat protein and the wild-type RNA operator have similar solution behaviour. Nucleic Acids Res. 2000;28:489-97.

21. Adams SR, Campbell RE, Gross LA, Martin BR, Walkup GK, Yao Y, et al. New biarsenical ligands and tetracysteine motifs for protein labeling in vitro and in vivo: Synthesis and biological applications. J Am Chem Soc. 2002;124:6063-76.

22. Stano P. Gene Expression Inside Liposomes: From Early Studies to Current Protocols. Chem - A Eur J [Internet]. Wiley-VCH Verlag; 2019 [cited 2021 Jan 20];25:7798-814. Available from: https://onlinelibrary.wiley.com/doi/abs/10.1002/chem.201806445

23. Dudley QM, Karim AS, Jewett MC. Cell-free metabolic engineering: Biomanufacturing beyond the cell. Biotechnol J. 2015;10:69-82.

24. Shimizu Y, Kuruma Y, Ying BW, Umekage S, Ueda T. Cell-free translation systems for protein engineering. FEBS J. 2006;273:4133-40.

25. Shimizu Y, Inoue A, Tomari Y, Suzuki T, Yokogawa T, Nishikawa K, et al. Cell-free translation reconstituted with purified components. Nat Biotechnol. 2001;19:751-5.

26. Harbers M. Wheat germ systems for cell-free protein expression. FEBS Lett [Internet]. Federation of European Biochemical Societies; 2014;588:2762-73. Available from:

http://linkinghub.elsevier.com/retrieve/pii/S0014579314004712

27. Kovtun O, Mureev S, Jung W, Kubala MH, Johnston W, Alexandrov K. Leishmania cell-free protein expression system. Methods [Internet]. Elsevier Inc.; 2011;55:58-64. Available from: 
http://dx.doi.org/10.1016/j.ymeth.2011.06.006

28. Ezore T, Suzuki T, Higashide S, Shintani E, Endo K, Kobayashi SI, et al. Cell-free protein synthesis system prepared from insect cells by freeze-thawing. Biotechnol Prog. 2006;22:1570-7.

29. Beckler GS, Thompson D, Van Oosbree T. In vitro translation using rabbit reticulocyte lysate. Methods Mol Biol. 1995;37:215-32.

30. Khan P, Aufdembrink LM, Engelhart AE. Isothermal SARS-CoV-2 Diagnostics: Tools for Enabling Distributed Pandemic Testing as a Means of Supporting Safe Reopenings. ACS Synth Biol [Internet]. American Chemical Society; 2020 [cited 2021 Oct 17];9:2861-80. Available from:

https://pubs.acs.org/doi/full/10.1021/acssynbio.0c00359

31. Aufdembrink LM, Khan P, Gaut NJ, Adamala KP, Engelhart AE. Highly specific, multiplexed isothermal pathogen detection with fluorescent aptamer readout. bioRxiv [Internet]. Cold Spring Harbor Laboratory; 2020 [cited 2020 May 10];2020.02.18.954719. Available from: https://doi.org/10.1101/2020.02.18.954719

32. Kopniczky MB, Canavan C, Mcclymont DW, Crone MA, Suckling L, Goetzmann B, et al. Cell-Free Protein Synthesis as a Prototyping Platform for Mammalian Synthetic Biology. 2020;

33. Carlson ED, Gan R, Hodgman CE, Jewett MC. Cell-free protein synthesis: Applications come of age. Biotechnol Adv [Internet]. Elsevier Inc.; 2012;30:1185-94. Available from: http://dx.doi.org/10.1016/j.biotechadv.2011.09.016

34. Gregorio NE, Levine MZ, Oza JP. A User's Guide to Cell-Free Protein Synthesis. Methods Protoc [Internet]. Multidisciplinary Digital Publishing Institute (MDPI); 2019 [cited 2021 Oct 5];2:1-34. Available from: /pmc/articles/PMC6481089/

35. Abil Z, Danelon C. Roadmap to Building a Cell: An Evolutionary Approach. Front Bioeng Biotechnol. 2020;8:1-8.

36. Gaut NJ, Adamala KP. Reconstituting Natural Cell Elements in Synthetic Cells. Adv Biol. 2021;5:1-20. 
37. Jackson NAC, Kester KE, Casimiro D, Gurunathan S, DeRosa F. The promise of mRNA vaccines: a biotech and industrial perspective. npj Vaccines 202051 [Internet]. Nature Publishing Group; 2020 [cited 2021 Oct 5];5:1-6. Available from: https://www.nature.com/articles/s41541-020-0159-8

38. Kis Z, Kontoravdi C, Dey AK, Shattock R, Shah N. Rapid development and deployment of high-volume vaccines for pandemic response. J Adv Manuf Process [Internet]. John Wiley \& Sons, Ltd; 2020 [cited 2021 Oct 5];2:e10060. Available from: https://onlinelibrary.wiley.com/doi/full/10.1002/amp2.10060 


\section{Figure $x 1$}
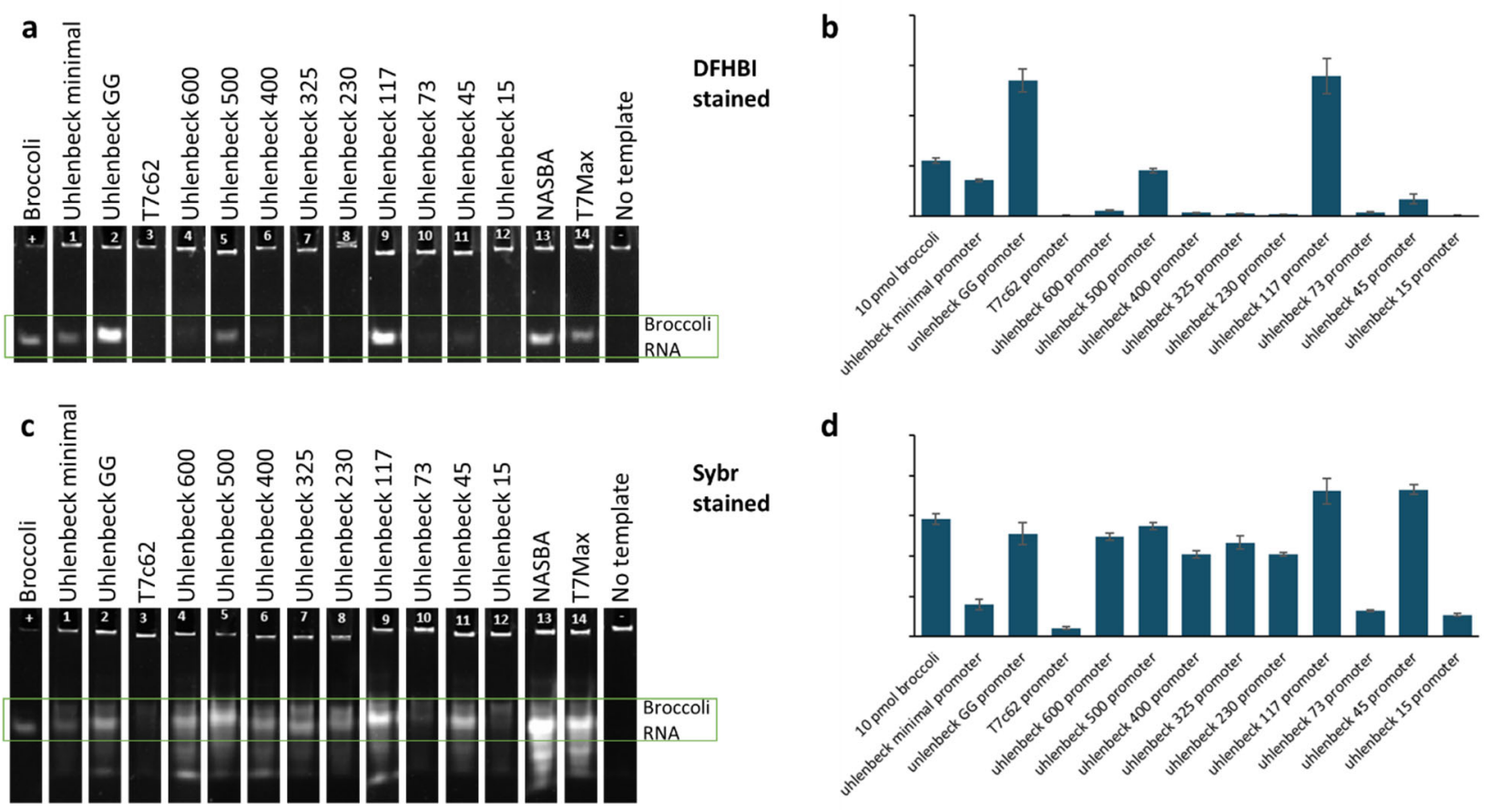

d
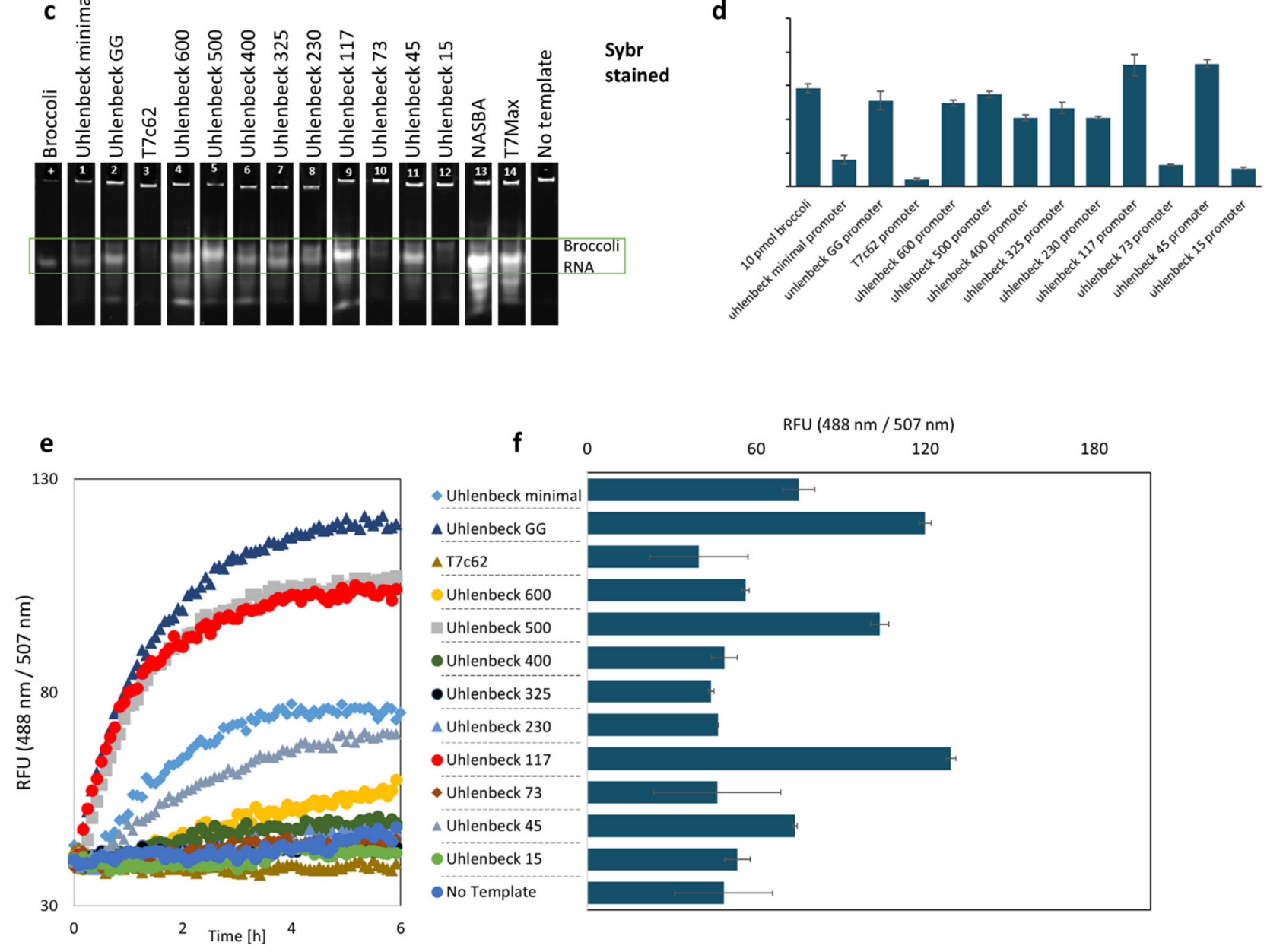

Figure x1: Testing different promoters in in-vitro transcription. a: transcription of the RNA broccoli

aptamer from linear dsDNA templates under different promoters. The gels are stained with DFHBI1T. $\mathbf{b}$ :

quantification of DFHBI1T stained gels. Y axis is the unitless relative brightness of the broccoli RNA band.

c: quantification of the same transcription gel as in a, stained with Sybr stain. d: quantification of the 
Sybr stained gel. The $Y$ axis is unitless relative brightness of the broccoli RNA band. e: time course of transcription from linear dsDNA broccoli aptamer templates with different promoters, one example trace for each experiment. The legend applies to panels e and f. f: end point fluorescence of broccoli RNA aptamer for 3 replicates for transcriptions showed on panel e, error bars are standard deviation. 


\section{Figure $x 2$}

a

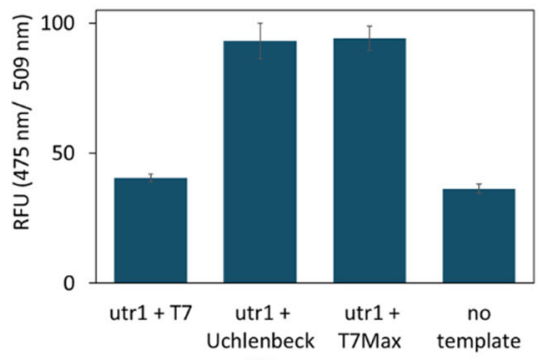

117

C

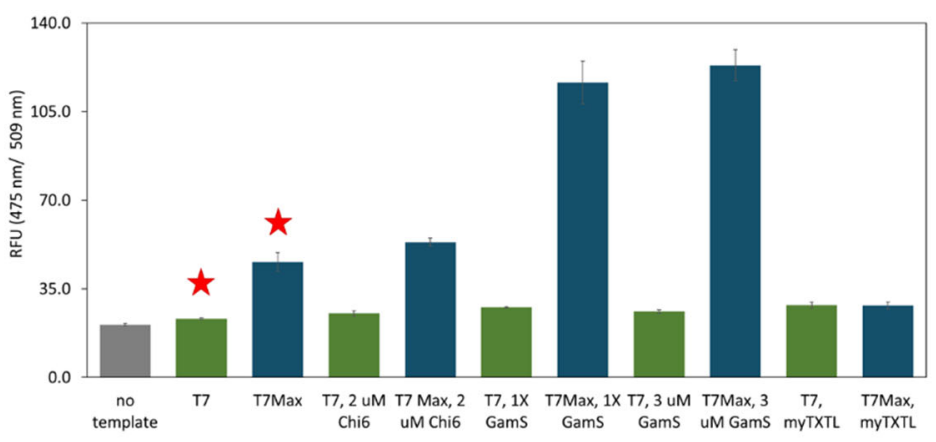

b

ర

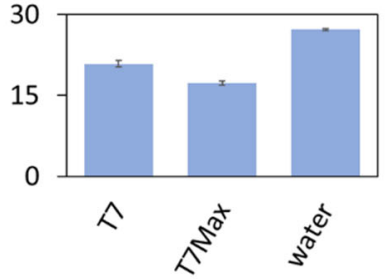

d T7 T7Max

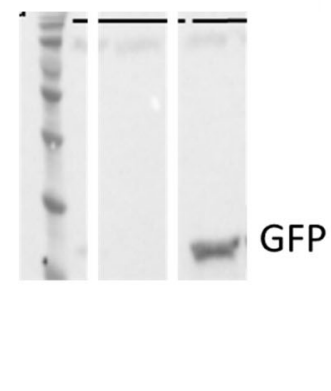

Figure x2: Cell-free TxTI of GFP from dsDNA linear template with different promoters. a: cell-free TxTI synthesis of eGFP, with two top candidate promoters, end point fluorescence measured after 8hour reactions. $\mathbf{b}$ : RT-qPCR measurement of mRNA abundance in TxTI GFP translation of classic T7 promoter, new T7 Max promoter, and no template control sample. Samples were collected after an 8 hour TxTI reaction. c: cell-free TxTI synthesis of GFP, T7 promoter (green bars) and T7Max promoter (blue bars), in house -made bacterial TxTI, with different ways of protecting linear DNA templates, and with commercially available myTXTL kit; end point fluorescence measured after 8 -hour reactions. For panels $\mathbf{a}, \mathbf{b}$ and $\mathbf{c}$ : each sample in triplicate, error bars are S.E.M. $\mathbf{d}$ : example of Western Blot analysis of GFP translation, 8 -hour end point translation from linear dsDNA template in home-made TXTL without DNA protection reagents (samples represent conditions showed on panel c marked with red star). 


\section{Figure $\times 3$}

a

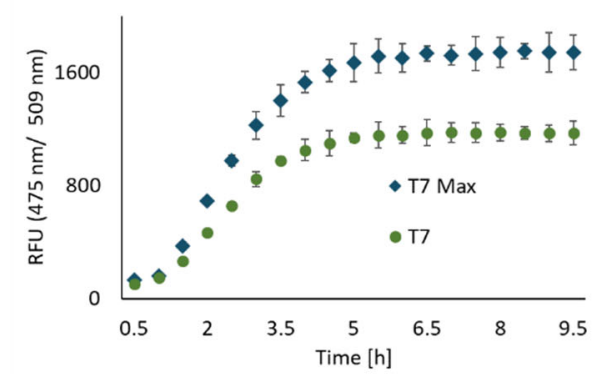

C

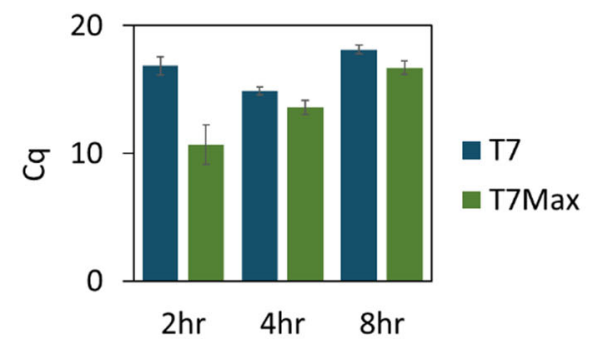

b

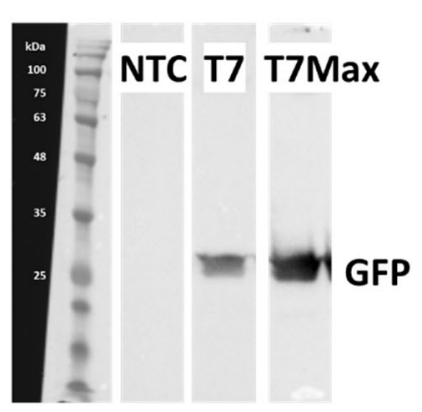

d

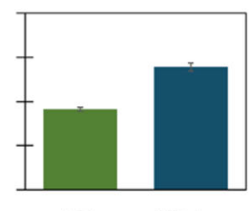

T7 T7Max

Figure x3: Cell-free TxTI of GFP from dsDNA circular plasmid template with different promoters. a:

time course expression of GFP under the classic T7 vs T7Max promoter. b: Western Blot analysis of expression of GFP. c: RT qPCR cycle (Cq) value quantifying abundance of GFP mRNA. d: quantification of Western Blots of GFP expression, expressed as unitless relative brightness value. All samples in triplicate, error bars represent S.E.M. Protein product was measured by end point measurements after an 8 -hour reaction. 
Figure $x 4$
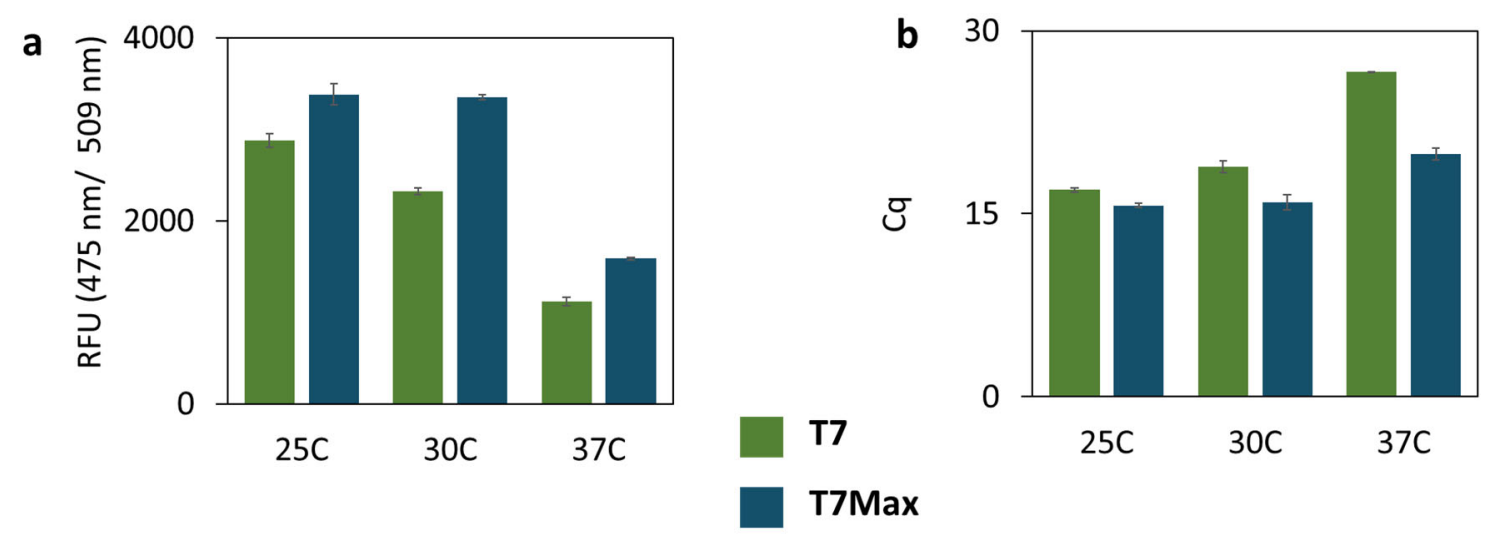

C
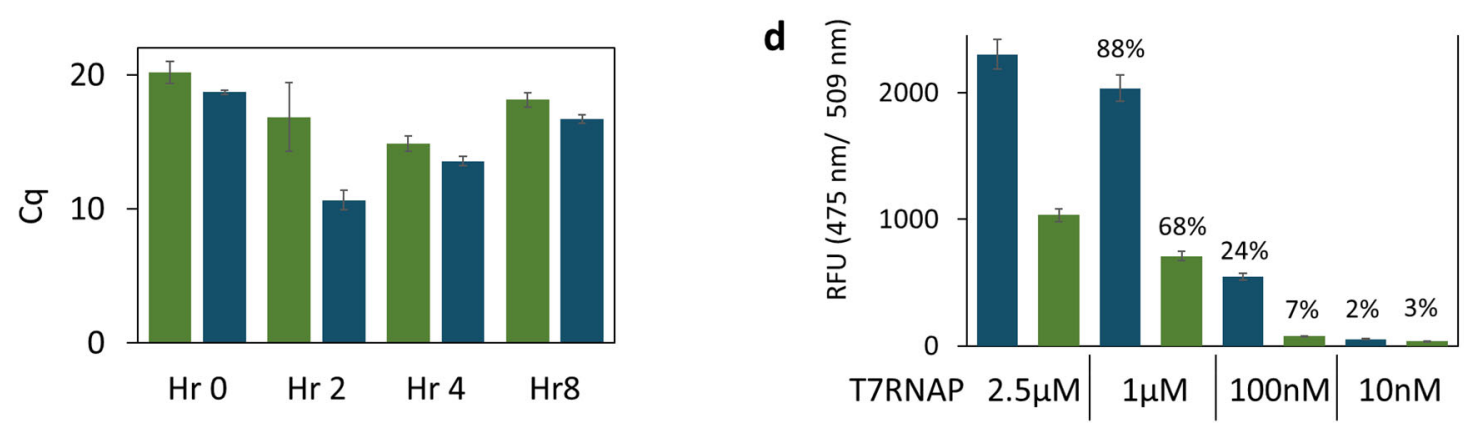

Figure x4: T7Max performance characterization. Translation of GFP protein from circular plasmid template was measured at different temperatures and with different T7 RNA polymerase concentration. All green bars: T7 promoter, all blue bars: T7Max promoter. a: expression of GFP measured after an 8hour reaction at different temperatures. b: RT qPCR measuring abundance of GFP mRNA in samples from panel a. c: mRNA abundance measured at different times during the TxTI reaction at $30^{\circ} \mathrm{C} . \mathbf{d}$ : expression of GFP measured after an 8 hour reaction with different concentration of T7 RNA polymerase. The percentage numbers above bars show fluorescence relative to the value at $2.5 \mu \mathrm{M} \mathrm{T7}$ RNAP for each promoter. All samples in triplicate, error bars represent S.E.M. 


\section{Figure $\times 5$}
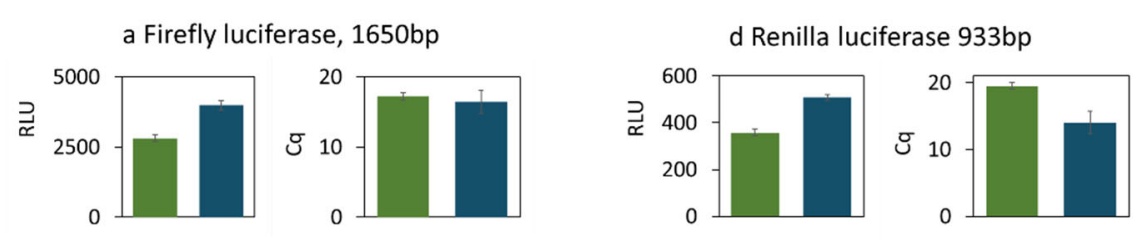

g PP7 monomer, 399bp

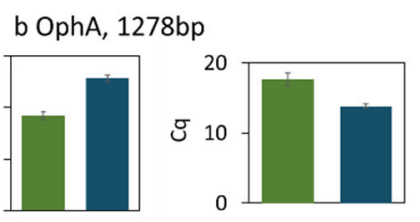

e Kanamycin resistance, $852 \mathrm{bp}$
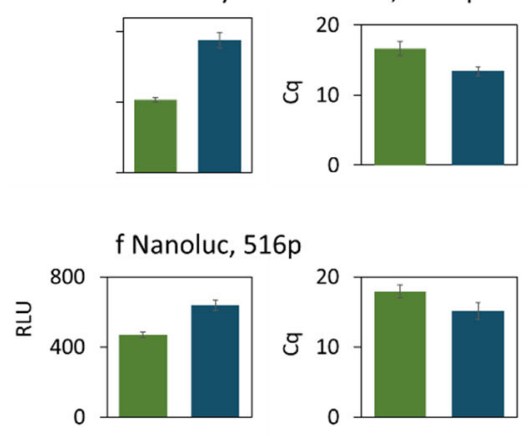

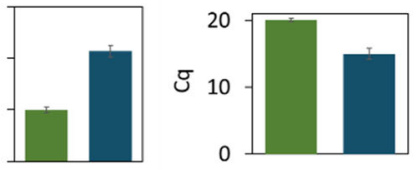

h MS2 monomer, 375bp
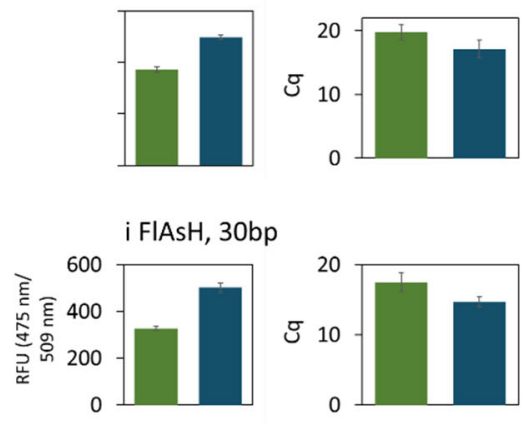

Figure x5: Performance of T7Max vs T7 promoter in different template lengths. All green bars: T7

promoter, all blue bars: T7Max promoter. Circular plasmid DNA template expression of proteins with different length of the open reading frame, from 1650 base pairs to 30 base pairs. Each graph shows protein product quantification and corresponding RT qPCR cycle (Cq) value quantifying abundance of mRNA for each protein. All samples in triplicate, error bars represent S.E.M. Protein product was measured by end point measurements after an 8 - hour reaction. Luminescence with appropriate luciferase product was used on panels $\mathbf{a}, \mathbf{d}, \mathbf{f}$. Quantification of appropriate size Western Blot band, expressed as unitless relative brightness value, was used on panels $\mathbf{b}, \mathbf{e}, \mathbf{g}$ and $\mathbf{h}$. Fluorescence with the arsenic ligand was measured on panel i. 


\section{Figure $\times 6$}

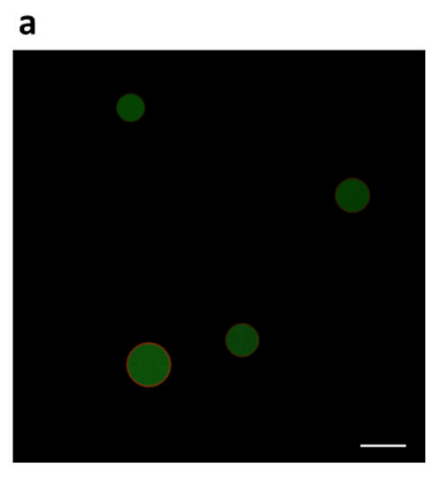

C

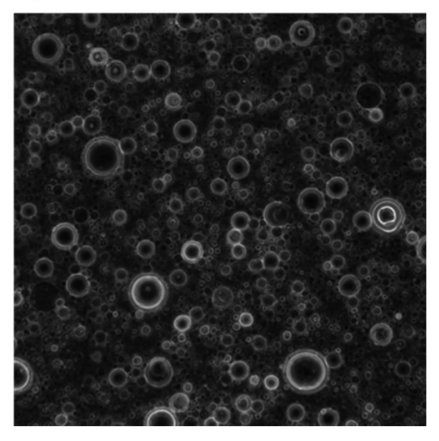

b

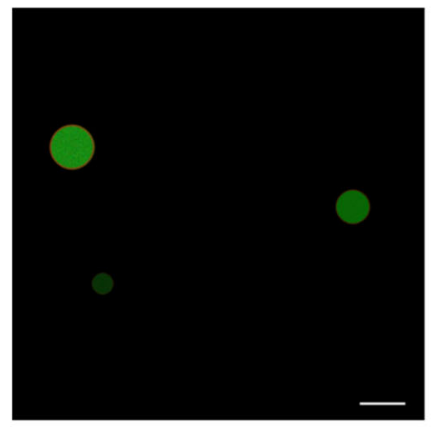

d

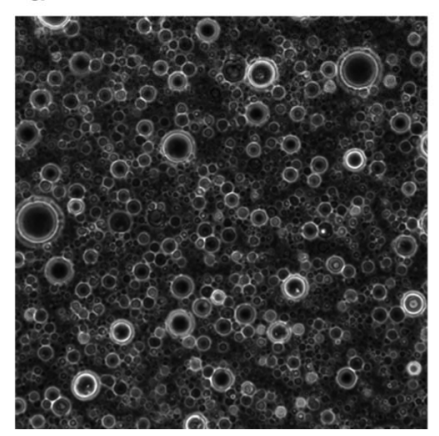

e

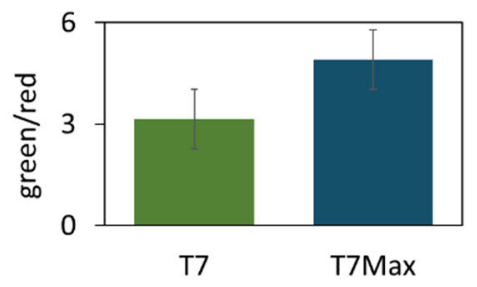

Figure x6: Synthetic minimal cells expressing GFP protein. Microscope images showing liposomes

encapsulating plasmid encoding GFP under T7 (panels a and c) and T7Max (panels b and d) promoters.

Panels $\mathbf{a}$ and $\mathbf{b}: 0.1 \mathrm{mM}$ lipid concentration, green (GFP) and red (rhodamine membrane dye) channels overlayed. Panels $\mathbf{c}$ and $\mathbf{d}$ : bright field showing density of liposomes at $10 \mathrm{mM}$ lipid. Scale bar is $5 \mu \mathrm{m}$. e: quantification of 5 images taken from different fields of view in samples at $10 \mathrm{mM}$ lipid; the value is ratio of total fluorescence in green channel to total fluorescence in red channel. Error bars represent S.E.M. 


\section{Figure $x 7$}
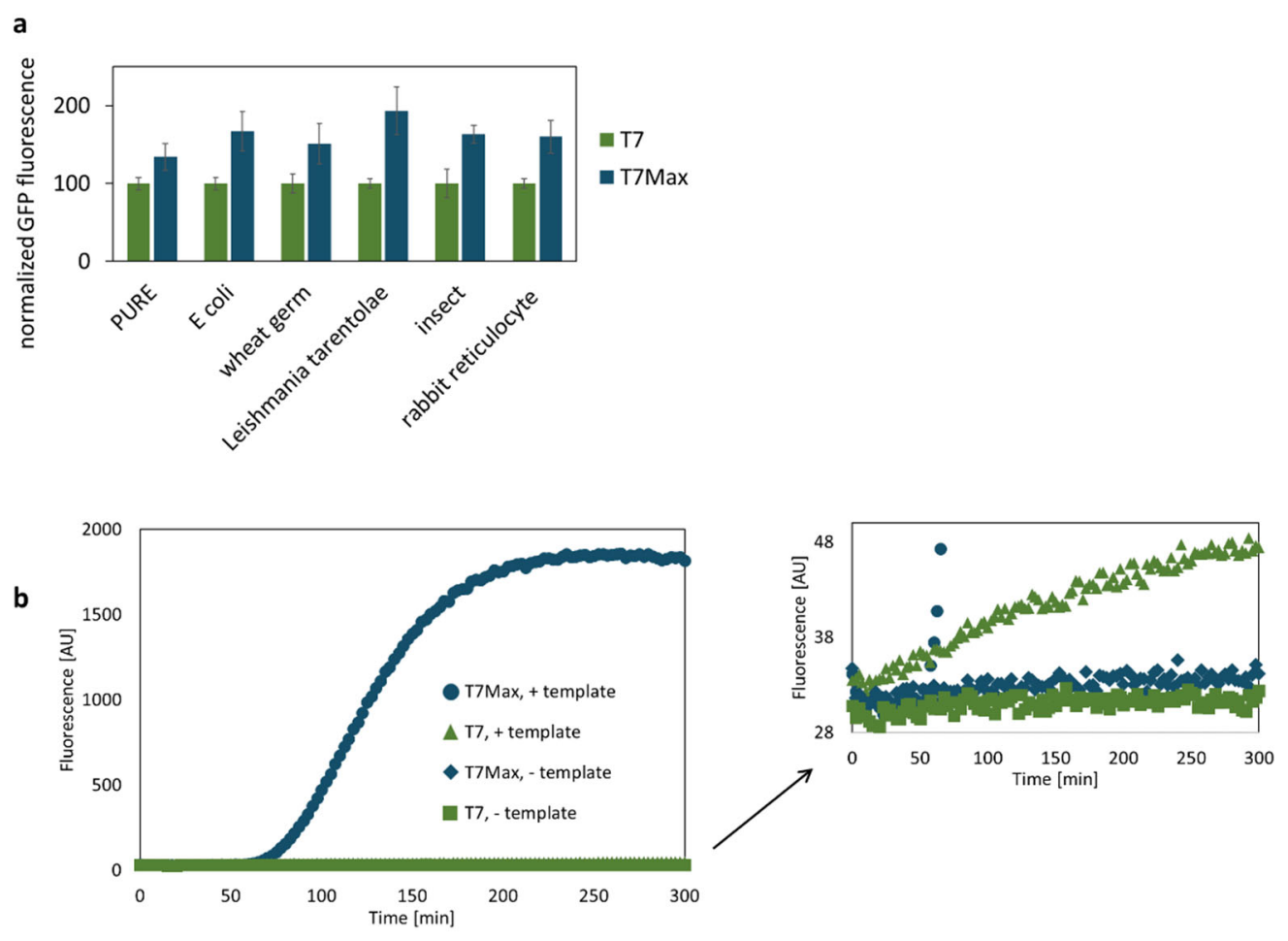

Figure x7: Performance of T7Max in different systems. a: Cell-free translation reactions based on different organisms. GFP plasmids were prepared for each specific commercial cell-free expression system (except $E$. Coli, which used the same plasmids as tested earlier, and in house made cell-free expression system). Fluorescence of GFP protein was measured after each reaction, and raw fluorescence was normalized so that classic T7 promoter fluorescence was assigned value 100, and T7Max sample fluorescence was scaled proportionally. All samples are in triplicate, error bars represent S.E.M. b: b: Apta-Nucleic Acid Sequence Based Amplification reaction detecting E. Coli gene, aggR. Reactions are identical except for the incorporation of T7Max vs classic T7 promoter. Fluorescence of the broccoli aptamer was measured every 2.5 minutes, excitation: $472 \mathrm{~nm}$ and emission: $507 \mathrm{~nm}$. All samples were performed in triplicate, and traces represent the average. 


\section{Table 1}

SAMPLE

SENSE STRAND
Promoter Sequence

ID

\begin{tabular}{c|ll}
\hline $\mathbf{1}$ & Uhlenbeck minimal promoter & TAATACGACTCACTATA \\
$\mathbf{2}$ & Uhlenbeck GG promoter & TAATACGACTCACTATAGG \\
$\mathbf{3}$ & T7c62 promoter & TAATACGACTCACAATCGCGGAG \\
$\mathbf{4}$ & Uhlenbeck 600 promoter & TAATACGACTCACTATAGGGATC \\
$\mathbf{6}$ & Uhlenbeck 500 promoter & TAATACGACTCACTATAGGGAGA \\
$\mathbf{7}$ & Uhlenbeck 325 promoter & TAATACGACTCACTATAGGGCTC \\
$\mathbf{8}$ & Uhlenbeck 230 promoter & TAATACGACTCACTATAGAGACT \\
$\mathbf{9}$ & Uhlenbeck 117 promoter & TAATACGACTCACTATAGGGAAG \\
$\mathbf{1 0}$ & Uhlenbeck 73 promoter & TAATACGACTCACTATAGCATCA \\
$\mathbf{1 1}$ & Uhlenbeck 45 promoter & TAATACGACTCACTATAGGACAT \\
$\mathbf{1 2}$ & Uhlenbeck 15 promoter & TAATACGACTCACTATACGATCA \\
$\mathbf{1 3}$ & NASBA promoter & T7Max Promoter \\
$\mathbf{1 4}$ & AATTTAATACGACTCACTATAGGGA \\
& AATTCTAATACGACTCACTATAGGGA \\
\hline
\end{tabular}

